\title{
Terson Syndrome in Aneurysmal Subarachnoid Hemorrhage: A Case Report
}

\author{
Sang-Hee Lee, MD, Jeong-Hwan Seo, MD, PhD, Sung-Hee Park, MD, PhD, \\ Yu Hui Won, MD, Myoung-Hwan Ko, MD, PhD
}

\begin{abstract}
Department of Physical Medicine and Rehabilitation, Research Institute of Clinical Medicine of Chonbuk National University-Biomedical Research Institute of Chonbuk National University Hospital, Jeonju, Korea
\end{abstract}

\begin{abstract}
Terson syndrome refers to oculocerebral syndrome of retinal and vitreous hemorrhage associated with spontaneous subarachnoid hemorrhage or all forms of intracranial bleeding. Recent observations have indicated that patients with spontaneous subarachnoid hemorrhage have an $18 \%$ to $20 \%$ concurrent incidence of retinal and vitreous hemorrhages with about $4 \%$ incidence of vitreous hemorrhage alone. Clinical ophthalmologic findings may have significant diagnostic and prognostic value for clinicians. Here we report a 45 -year-old female patient who suffered from blurred vision after subarachnoid hemorrhage. She was diagnosed as Terson syndrome. After vitrectomy, she recovered with normal visual acuity which facilitated the rehabilitative process. We also performed visual evoked potentials to investigate abnormalities of visual dysfunction. Based on this case, we emphasize the importance of early diagnosis of Terson syndrome.
\end{abstract}

Keywords Terson syndrome, Visual evoked potentials, Subarachnoid hemorrhage

\section{INTRODUCTION}

Terson syndrome refers to vitreous hemorrhage stemming from raised intracranial pressure associated with subarachnoid or subdural hemorrhage [1]. Intraocular hemorrhage occurs in $20 \%$ of patients with subarachnoid hemorrhage. However, vitreous hemorrhage only affects $4 \%$ of patients with subarachnoid hemorrhage [2].

Received June 3, 2014; Accepted September 15, 2014

Corresponding author: Myoung-Hwan Ko

Department of Physical Medicine and Rehabilitation, Research Institute of Clinical Medicine of Chonbuk National University-Biomedical Research Institute of Chonbuk National University Hospital, 20 Geonjiro, Deokjin-gu, Jeonju 561-712, Korea

Tel: +82-63-250-1795, Fax: +82-63-254-4145, E-mail: mhko@jbnu.ac.kr

(c) This is an open-access article distributed under the terms of the Creative Commons Attribution Non-Commercial License (http://creativecommons. org/licenses/by-nc/4.0) which permits unrestricted noncommercial use, distribution, and reproduction in any medium, provided the original work is properly cited.

Copyright $\odot 2015$ by Korean Academy of Rehabilitation Medicine
In most cases, vitreous hemorrhage is naturally cleared and vision is restored within a year. However, clearing of hemorrhage can take several years in some cases. Such delayed clearing can be complicated by epiretinal membrane. If left untreated, such conditions can lead to loss of vision or serious visual impairment [3]. Therefore, it should be clarified whether the visual impairment stems from brain lesions or from vitreous hemorrhage associated with raised intracranial pressure. If the etiology is identified as vitreous hemorrhage, the standing blood in the vitreous should be removed. Complications such as epiretinal membrane, retinal detachment, and macular degeneration should be appropriately treated for better prognosis. Patients with subarachnoid hemorrhage or subdural hemorrhage often present cognitive impairment which hinders accurate diagnosis [1].

We encountered a case of aneurysmal subarachnoid hemorrhage accompanied by vitreous hemorrhage of the 
right eye that led to visual impairment. Early diagnosis of vitreous hemorrhage and subsequent vitrectomy were carried out based on ophthalmologic findings and visual evoked potentials (VEPs). In our case, the treatment resulted in recovery of vision and improved activities of daily living.

\section{CASE REPORT}

A 45-year-old female patient developed aneurysmal subarachnoid hemorrhage on March 28, 2014. She received craniotomy, cerebral aneurysm clipping, and external ventricular drainage. She was transferred to Rehabilitation Medicine Department on April 20, 2014.
At the time of transfer, she was fully conscious. However, she scored only 15 on Korean Mini-Mental State Examination (K-MMSE), indicating cognitive impairment. In the Medical Research Council Manual Muscle Test, the patient was found to have grade 4 in the upper and lower limbs of the right side and grade 3 in those of the left. She scored 76 on Korean Modified Barthel Index (K-MBI). She was able to roll over, sit and stand. She could ambulate with minimal assistance. Her eye movement, pupil size, and reflex in both eyes were normal.

Visual acuity of both eyes measured 2 days after the transfer due to the complaint of decreased vision in the right eye was $30 \mathrm{~cm}$ in the right eye on finger counting and 0.6 in the left eye. Brain computed tomography (CT)
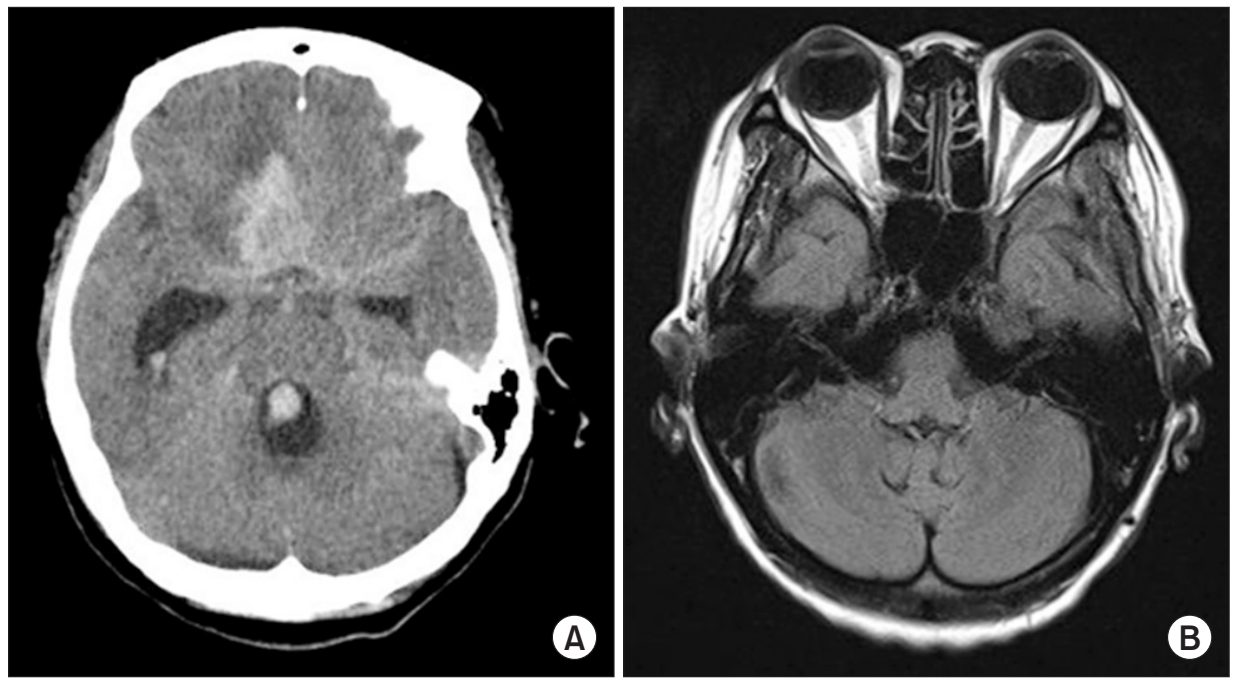

Fig. 1. Brain computed tomography at the time of admission showing subarachnoid hemorrhage after aneurysmal rupture (A) and brain magnetic resonance imaging (FLAIR image) obtained after right blindness revealing normal finding in right eyeball, suggesting intravitreous bleeding (B).

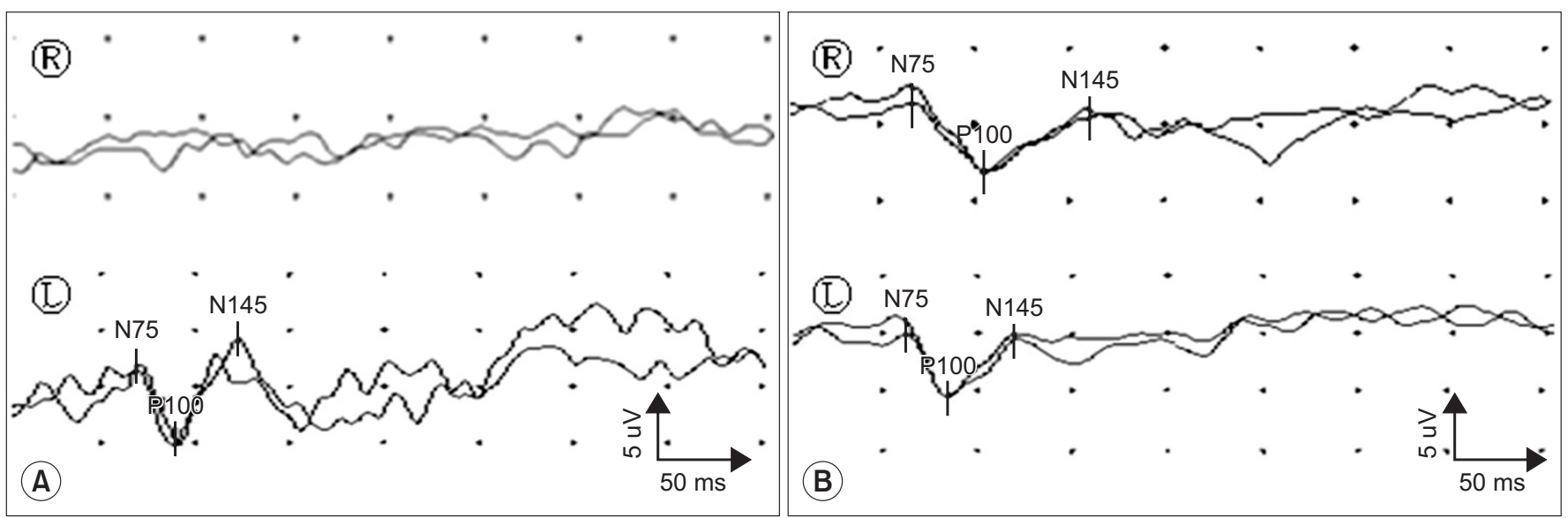

Fig. 2. Latencies of N75, P100, N145 of pattern reversal visual evoked potential (VEP) in the patient with subarachnoid hemorrhage before vitrectomy (A) and after vitrectomy (B). VEP was not evoked in the right eye before vitrectomy. After vitrectomy, VEP showed relatively prolonged P100 latency and low amplitude in the right eye stimulation compared to the left eye stimulation. 

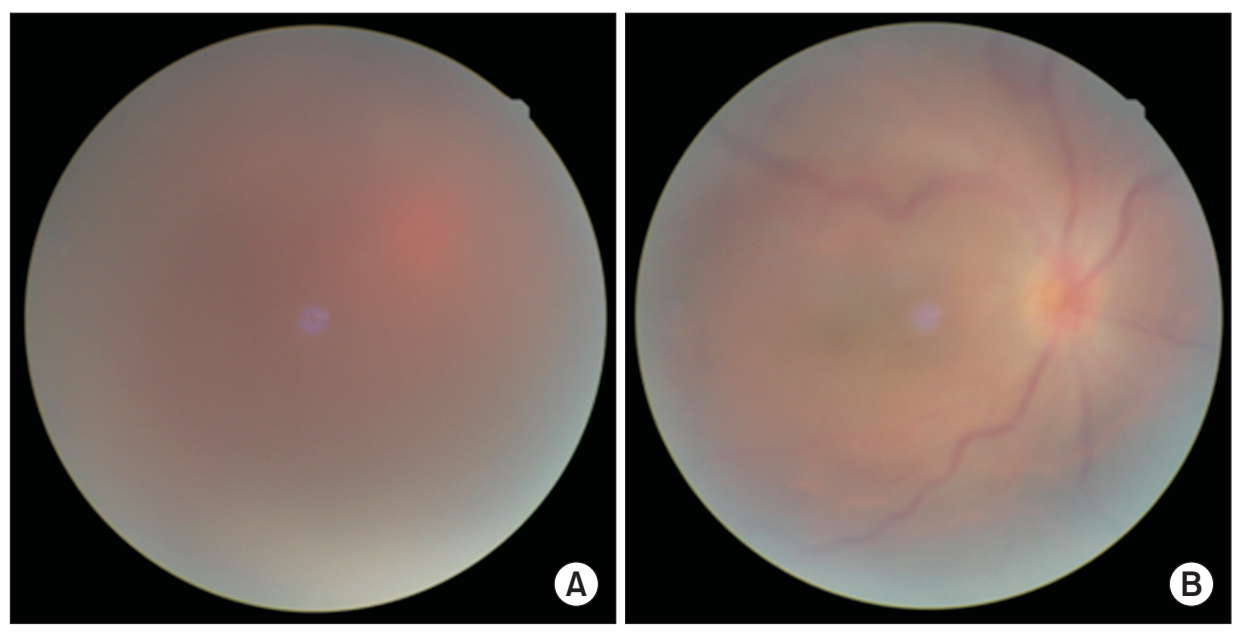

Fig. 3. Right fundoscopy showing intravitreous hemorrhage before vitrectomy (A) and normal finding after vitrectomy (B).

and magnetic resonance imaging (MRI) performed 2 days after the transfer (April 22, 2014) showed no abnormality in visual pathways (Fig. 1). However, VEPs was not evoked in the right eye (Fig. 2). Fundus examination and ocular ultrasound performed at our hospital's Department of Ophthalmology showed vitreous hemorrhage in the right eye (Fig. 3).

Vitrectomy of the right eye was performed on April 24, 2014 at our hospital. Optic discs and macula were observed on the fundus examination performed a day after the surgery. Visual acuity measured in both eyes was 0.15 in the right eye and 0.6 in the left. Following vision recovery, the patient showed rapid improvement in her activities of daily living including ambulation. Her visual acuity measured at the time of discharge (May 16, 2014) was improved to 0.8 in the right eye and 1.0 in the left. Retina was normal on fundus examination (Fig. 3). Her right eye showed no evoked potentials after transfer. However, the evoked potentials were recovered on VEPs performed right before discharge (Fig. 2). Compared to the healthy left eye, P100 latency was delayed in the right eye with amplitude reduced by $40 \%$. The patient scored 87 on KMBI. She could ambulate independently and perform activities of daily living.

\section{DISCUSSION}

Terson syndrome, a type of vitreous hemorrhage that can occur concurrently with arachnoid hemorrhage or subdural hemorrhage arising from trauma, hypertension, or ruptured aneurysm is difficult to be diagnosed due to patient's impaired cognitive ability. The case reported here was also accompanied with cognitive impairment as evidenced by 15 points on MMSE. She could not describe her visual impairment clearly. She suffered difficulty in activities of daily living. Patients with brain disease and cognitive impairment are unable to clearly communicate their visual problems in the rehabilitative process. They often express their inability to perform tasks through simply refusing to do them. Therefore, when patients are unable to perform tasks during rehabilitation therapy due to intracranial lesions (especially if raised intracranial pressure causes subarachnoid hemorrhage or subdural hemorrhage), one should pay close attention to whether such difficulties stem from visual impairment. Ophthalmological examinations should be carried out to accurately identify the etiology of visual impairment before attributing it to brain lesion alone.

One study reported that the incidence of vitreous hemorrhage was $53 \%$ in patients with loss of consciousness due to subarachnoid hemorrhage [4]. That study has noted that the incidence of vitreous hemorrhage in patients with temporary or long-term coma is higher [4]. Therefore, diagnostic tests for screening Terson syndrome are very important. Terson syndrome can be confirmed by ophthalmological examinations, such as fundus examination and ocular ultrasonography. Half-moon shaped hyperintensity of retina on CT and MRI has been reported to have $66.7 \%$ sensitivity for diagnosing Terson syndrome [5]. Unlike previous case reports, the case reported here did not show abnormalities on CT or brain MRI. Studies on Terson syndrome discussed ophthalmological and diagnostic imaging as well as vision recovery. Very few cases reported the usefulness of VEPs or success of task 
performance of recovered vision. One study reported that VEPs had low sensitivity for diagnosing Terson syndrome [6]. However, ocular problems in our case was suspected through VEPs. This study is different from previous ones in that post-surgical vision improvement was objectively assessed in this study.

The role of VEPs is to confirm the existence of sensory abnormality on history taking or neurologic examination or confirm the existence of sensory disturbance in patients with suspected but clinically unconfirmed demyelinating disease. VEPs are also helpful in identifying anatomical location of the disease and objectively assessing changes in patients over time [7]. VEPs are particularly useful in confirming Terson syndrome and in observing the outcome of patients with unconsciousness or cognitive impairment.

Visual impairment can negatively impact the ability of patient to perform activities of daily living and the recovery of stroke patients with hemiplegia $[8,9]$. For the patient reported in this case study, her post-stroke upper and lower limb muscle strength was preserved in good grade. However, Terson syndrome inhibited her activities of daily living, such as ambulation and stair gait that required at least moderate assistance. She also had impaired ability to move pegs, read, or write. Following diagnosis of Terson syndrome, she received vitrectomy and successfully recovered her vision. This immediately improved her activities of daily living. Vision improvement enabled a comprehensive rehabilitation therapy encompassing ambulation, upper limb function, linguistic, and cognitive therapy. At the time of discharge, she was able to perform most of the activities of daily living under supervision, demonstrating that visual impairment can affect all aspects of rehabilitation therapy.

Middleton et al. [9] have reported that early rehabilitation therapy using visual compensation techniques is beneficial in pre-surgery patients with peripheral impairment of vision due to Terson syndrome. Our case experienced recovery of vision through ophthalmological surgery immediately following diagnosis of Terson syndrome. Middleton et al. [9] have reported that rehabilitation therapy following diagnosis of Terson syndrome is successful without resorting to surgery. However, surgery was eventually needed to improve vision due to retinal detachment. The case in our study with cognitive and visual impairment was found through close monitoring and physical examination. She did not show any ocular abnormalities on brain MRI. However, her diagnosis Terson syndrome was confirmed through VEPs. Subsequent vitrectomy through consultation with ophthalmology brought recovery of vision which enabled successful rehabilitation therapy. Shaw and Landers [10] have reported that mortality has doubled with the existence of intraocular hemorrhage. Terson syndrome can be an important marker to assess patient's outcome. Vision restoration and lowered mortality can be achieved by early diagnosis and prognosis monitoring of intraocular bleeding through ophthalmological examinations and VEPs in patients with subarachnoid hemorrhage presenting cognitive impairment or visual field problems. This will greatly contribute to patients' activities of daily living.

In summary, we report a case of subarachnoid hemorrhage and visual impairment of the right eye arising from vitreous hemorrhage. VEPs and ophthalmological examinations enabled the early diagnosis of Terson syndrome which was treated by vitrectomy. The patient recovered her vision to the level of pre-hospitalization. Her ability to perform daily activities was also improved.

\section{CONFLICT OF INTEREST}

No potential conflict of interest relevant to this article was reported.

\section{ACKNOWLEDGMENTS}

This paper was supported by Fund of Biomedical Research Institute, Chonbuk National University Hospital.

\section{REFERENCES}

1. Kim US, Yu SY, Kwak HW. Incidence and postoperative visual outcome of Terson's syndrome. J Korean Ophthalmol Soc 2002;43:2451-6.

2. Timberlake WH, Kubik CS. Follow-up report with clinical and anatomical notes on 280 patients with subarachnoid hemorrhage. Trans Am Neurol Assoc 1952;56:26-30.

3. Shaw HE Jr, Landers MB, Sydnor CF. The significance of intraocular hemorrhages due to subarachnoid hemorrhage. Ann Ophthalmol 1977;9:1403-5.

4. Frizzell RT, Kuhn F, Morris R, Quinn C, Fisher WS 3rd. 
Screening for ocular hemorrhages in patients with ruptured cerebral aneurysms: a prospective study of 99 patients. Neurosurgery 1997;41:529-33

5. Swallow CE, Tsuruda JS, Digre KB, Glaser MJ, Davidson HC, Harnsberger HR. Terson syndrome: CT evaluation in 12 patients. AJNR Am J Neuroradiol 1998;19:743-7.

6. Wiethölter S, Steube D, Stotz HP. Terson syndrome: a frequently missed ophthalmologic complication in subarachnoid hemorrhage. Zentralbl Neurochir 1998;59:166-70.

7. Chiappa KH. Principle of evoked potentials. In: Chiappa $\mathrm{KH}$, editor. Evoked potentials in clinical medi- cine. 3rd ed. Philadelphia: Lippincott-Raven; 1977. p. 1-30.

8. Wade DT, Skilbeck CE, Hewer RL. Predicting Barthel ADL score at 6 months after an acute stroke. Arch Phys Med Rehabil 1983;64:24-8.

9. Middleton K, Esselman P, Lim PC. Terson syndrome: an underrecognized cause of reversible vision loss in patients with subarachnoid hemorrhage. Am J Phys Med Rehabil 2012;91:271-4.

10. Shaw HE Jr, Landers MB 3rd. Vitreous hemorrhage after intracranial hemorrhage. Am J Ophthalmol 1975;80:207-13. 